\title{
Concerning the Efficiency of Corrosion Inhibitors as Given by SVET
}

\author{
A.C. Bastos, ${ }^{*}$ M.L. Zheludkevich, M.G.S. Ferreira \\ DECV/CICECO, Universidade de Aveiro, 3810-193 Aveiro, Portugal
}

Received $21^{\text {st }}$ May 2007; accepted $17^{\text {th }}$ August 2007

\begin{abstract}
The possibility of obtaining corrosion inhibition efficiency from the Scanning Vibrating Electrode Technique (SVET) is investigated. Results are compared with those obtained by Electrochemical Impedance Spectroscopy (EIS) for the inhibition of iron and zinc with cerium nitrate and $1 \mathrm{H}$-benzotriazole. Examples of good and bad estimations are given and an interpretation for the discrepancies observed is advanced. Large deviations occur when corrosion products are formed since this leads to an underestimation of the currents measured by SVET. Visual inspection of the samples may identify the cases more susceptible to deviation. The approach discussed here can be used to obtain more quantitative information from SVET measurements but should not replace the traditional ways used to determine the inhibition efficiency.
\end{abstract}

Keywords: SVET, EIS, corrosion, inhibitor efficiency.

\section{Introduction}

The inhibition efficiency, $I E$, of a substance is estimated by comparing the corrosion rate in the presence and in the absence of that substance [1]:

$$
I E(\%)=\frac{\text { Rate uninhibited }- \text { Rate inhibited }}{\text { Rate uninhibited }} \times 100
$$

The rate can be determined by different ways. The loss of mass of metal samples, the concentration of metal ions in solution and the volume of $\mathrm{H}_{2}$ evolved in acidic media are directly proportional to the corrosion rate. The corrosion current, $i_{c o r r}$, is also a direct indication of the corrosion rate. It is determined by electrochemical methods, typically by extrapolation of Tafel lines or by the polarization resistance method. The inhibition efficiency is calculated from $i_{\text {corr }}$ using equation (2), where $i$ is the current of the sample in the inhibiting medium and $i_{0}$ is the current measured in the corrosive medium,

\footnotetext{
* Corresponding author. E-mail address: acbastos@cv.ua.pt
} 


$$
I E(\%)=\left(1-\frac{i}{i_{0}}\right) \times 100
$$

The $i_{\text {corr }}$ may also be determined by Electrochemical Impedance Spectroscopy (EIS). The charge transfer resistance, $R_{c t}$, obtained by EIS, is inversely proportional to the corrosion rate [2]. In this case, the inhibition efficiency can be determined by equation (3), where $R_{c t 0}$ is the charge transfer resistance in the corrosive medium and $R_{c t}$ is the charge transfer resistance in the inhibiting medium,

$$
I E(\%)=\left(1-\frac{R_{c t_{0}}}{R_{c t}}\right) \times 100
$$

A new technique to study corrosion is now available. The Scanning Vibrating Electrode Technique (SVET) has the ability to measure ionic fluxes in aqueous solutions which makes it suitable to study many corrosion systems, particularly those that show a clear separation of the regions where anodic and cathodic reactions take place. SVET was firstly used in the life sciences [3-5], being introduced later in the corrosion field [6-9]. So far, most SVET studies in corrosion focused on the evolution of the anodic and cathodic activities in space and time, with a straightforward comparison of the current magnitudes obtained in different samples. The present work explores and discusses the possibility of obtaining more quantitative information from the SVET measurements, namely, the efficiency of corrosion inhibitors. The inhibition of iron and zinc corrosion in $0.05 \mathrm{M} \mathrm{NaCl}$, using cerium (III) nitrate and $1 \mathrm{H}$-benzotriazole, is studied by SVET and EIS and the inhibition efficiency obtained by the two techniques is compared.

\section{Experimental}

\section{Materials and reagents}

The metal electrodes were made with pure iron $(99.5+\%)$ and pure zinc (99.95\%), both supplied by Goodfellow (UK). The corrosive medium was aqueous $0.05 \mathrm{M} \mathrm{NaCl}$ prepared with distilled water and p.a. grade reagent. Two corrosion inhibitors, one of organic nature (1H-benzotriazole, BTA, 99\%, Aldrich) and one of inorganic nature (cerium nitrate, 99\%, Aldrich) were added to the corrosive medium at a concentration of $0.5 \mathrm{~g} \mathrm{~L}^{-1}$.

\section{Electrochemical Impedance Spectroscopy}

Experimental set-up: a Femtostat FAS2/PCI4 (Gamry Instruments, USA) was employed, with a three electrode arrangement, using a saturated calomel electrode as reference, a $1 \mathrm{~cm}^{2}$ platinum counter electrode and $1 \mathrm{~cm}^{2}$ pure zinc or pure iron working electrodes. The measurements were made inside a Faraday cage at room temperature and solutions were kept unstirred and aerated. 
Experimental conditions: measurements were performed in the frequency range $100 \mathrm{kHz}-5 \mathrm{mHz}$, with 7 points per decade with logarithmic distribution, with an a.c. signal of $10 \mathrm{mV}$ rms at open circuit potential.

\section{Scanning Vibrating Electrode Technique}

The SVET equipment was manufactured by Applicable Electronics (USA). The probe had a spherical tip of $20 \mu \mathrm{m}$ and the vibration amplitude was $20 \mu \mathrm{m}$. Scan maps comprised $100 \times 50$ points and were obtained with the probe vibrating 200 $\mu \mathrm{m}$ above the surface.

a
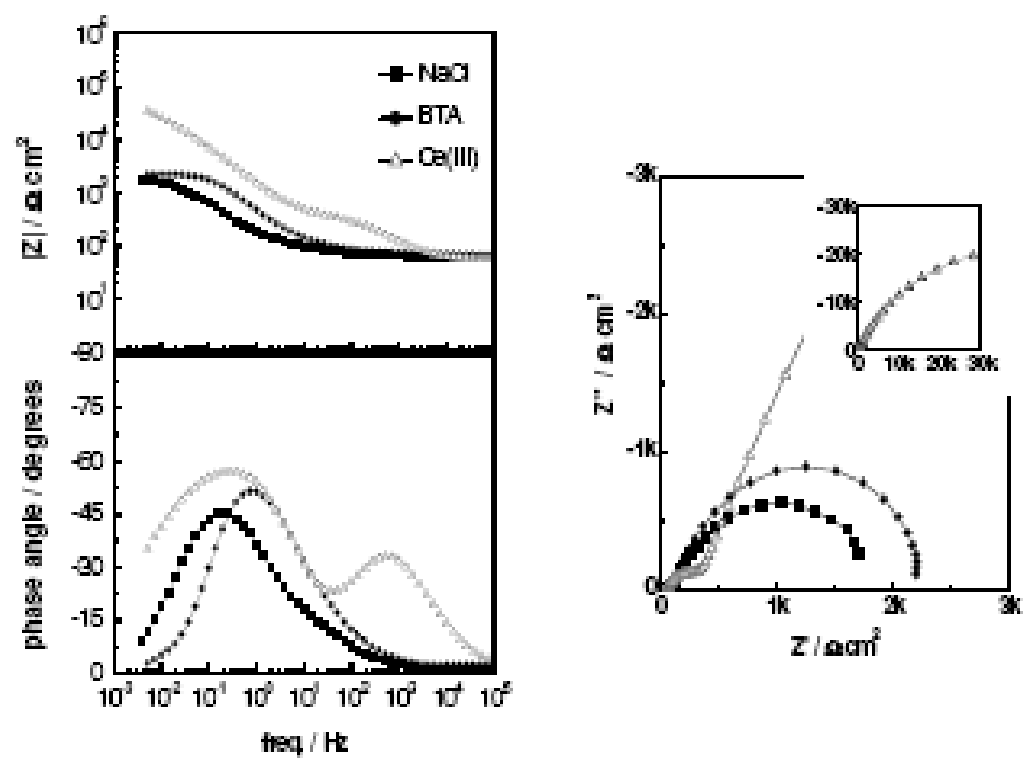

$\mathrm{b}$
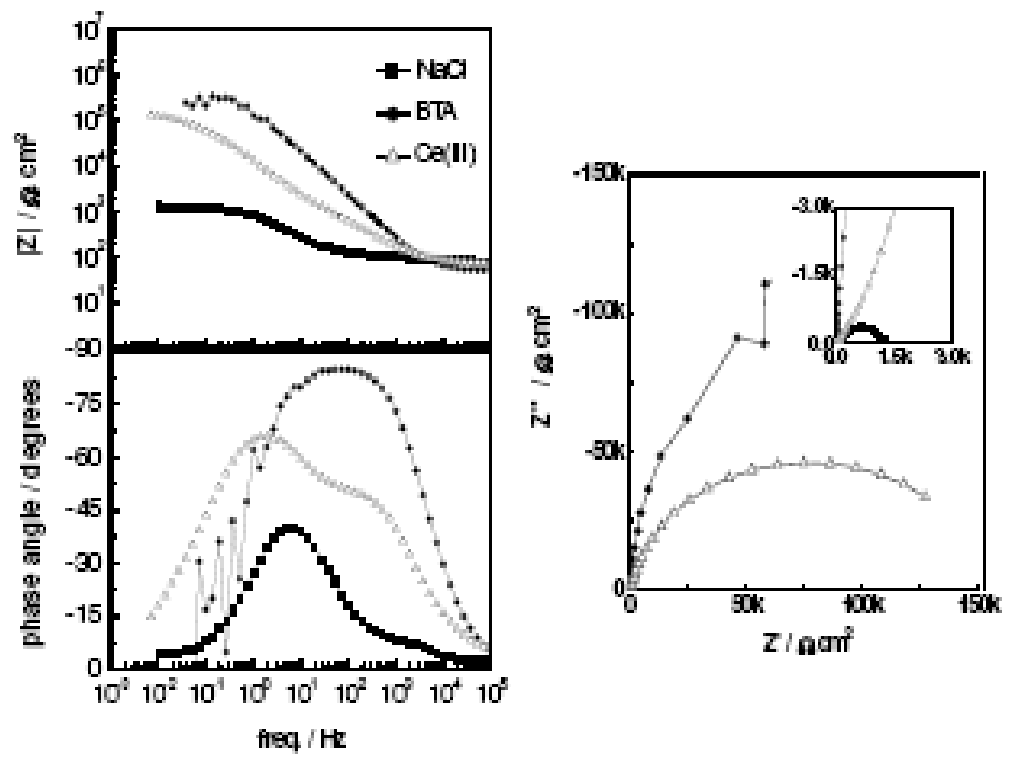

Figure 1. Bode plots (left) and complex plane representations (right) of the impedance of $\mathrm{Fe}(\mathbf{a})$ and $\mathrm{Zn}(\mathbf{b})$ after 24 hours of immersion in the corrosive medium $(0.05 \mathrm{M}$ $\mathrm{NaCl}$ ) and in the two inhibitive solutions: $0.05 \mathrm{M} \mathrm{NaCl}+0.5 \mathrm{~g} \mathrm{~L}^{-1} \mathrm{Ce}\left(\mathrm{NO}_{3}\right)_{3}$ and 0.05 $\mathrm{M} \mathrm{NaCl}+0.5 \mathrm{~g} \mathrm{~L}^{-1}$ benzotriazole. 


\section{Results}

EIS

Fig. 1 gives the impedance, in Bode and complex plane representations, of Fe and $\mathrm{Zn}$ after 24 hours of immersion in the corrosive medium and in the two inhibiting solutions. The impedance spectra of iron - Fig. 1 a) - in $\mathrm{NaCl}$ and BTA show a single time constant, attributed to the response of the double layer capacitance, $C_{d l}$, and the charge transfer resistance, $R_{c t}$. $C_{d l}$ is smaller for BTA which is explained by the adsorption of inhibitor to the metal surface that decreases the active area [10]. For the same reason, $R_{c t}$ is slightly higher in BTA than in $\mathrm{NaCl}$. When iron is immersed in Ce(III), a second time constant appears at higher frequencies, being it attributed to the formation of a passive layer of cerium oxides/hydroxides on the metal surface. In this solution, $C_{d l}$ is much smaller and $R_{c t}$ much higher compared to the other two media. The pure resistive response of $R_{c t}$ was not observed. The detection of $R_{c t}$ would need measurements at lower frequencies.

The impedance of zinc in the two inhibiting media after 24 hours - Fig. $1 \mathrm{~b}$ ) - is much higher than in the corrosive medium, confirming the high efficiency of these inhibitors for the protection of zinc. In BTA, a single time constant response is detected, being it attributed, as for iron, to the $C_{d l}$ and $R_{c t}$ of zinc exposed in areas not covered by adsorbed BTA. In Ce(III), a two time constant response is observed. As for iron in the same solution, the high frequency response is attributed to a passive layer due to precipitation of $\mathrm{Ce}(\mathrm{III})$ hydroxide/oxide on the metal surface [11]. At lower frequencies appears the response of $C_{d l}$ and $R_{c t}$ on the zinc not covered by the passive layer.

The values of $R_{c t}$ for iron and zinc in the three solutions were determined by numerical fitting using a commercial software package and are presented in Table 1 along with the inhibition efficiency calculated by equation 3 .

Table 1. Values of $R_{c t}$ and corresponding inhibition efficiency for iron and zinc in the three solutions tested.

\begin{tabular}{cccc}
\hline Metal & Solution & $R_{c t}\left(\Omega \mathrm{cm}^{2}\right)$ & $I E(\%)$ \\
\hline $\mathrm{Fe}$ & $\mathrm{NaCl}$ & $2.13 \times 10^{3}$ & 0 \\
& $\mathrm{BTA}$ & $2.46 \times 10^{3}$ & 13.4 \\
& $\mathrm{Ce}$ & $5.54 \times 10^{4}$ & 96.2 \\
$\mathrm{Zn}$ & $\mathrm{NaCl}$ & $1.14 \times 10^{3}$ & 0 \\
& $\mathrm{BTA}$ & $2.81 \times 10^{5}$ & 99.6 \\
& $\mathrm{Ce}$ & $1.43 \times 10^{5}$ & 99.2 \\
\hline
\end{tabular}

The SVET detects the electric fields in solution associated with the ionic currents generated at the metal surface [7]. A prior calibration permits to calculate the local current densities from the potential differences measured in solution [8]. Usually positive currents correspond to anodic activity and negative currents to cathodic processes. Fig. 2 shows images and current maps obtained in solution, above zinc and iron electrodes after 24 hours of immersion in the three solutions. 
a)
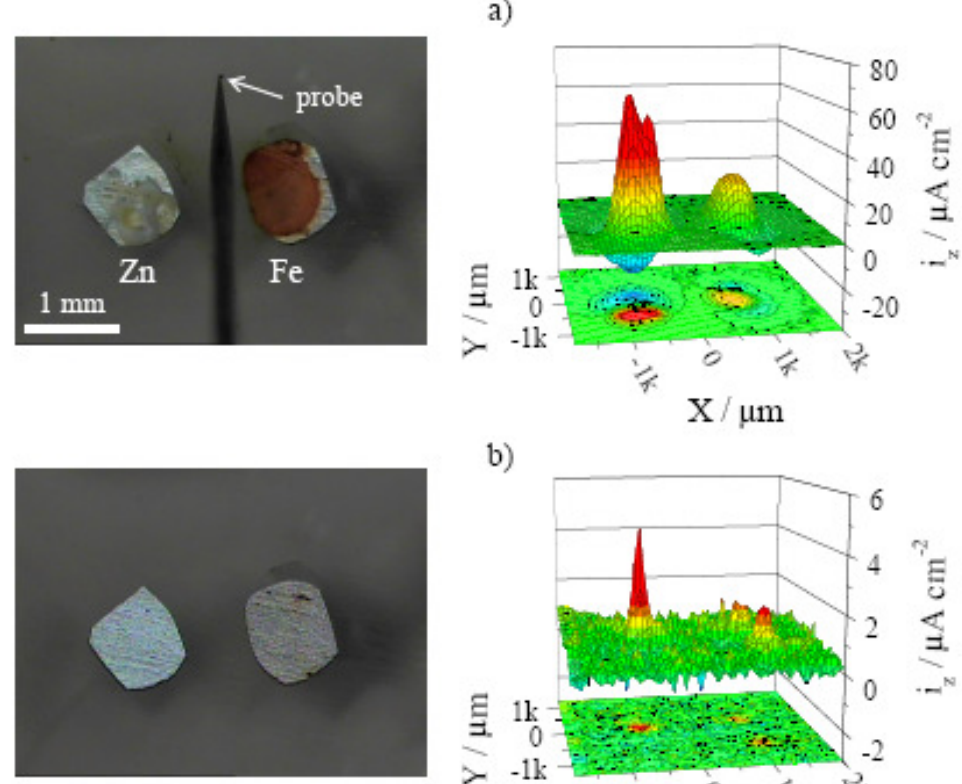

b)
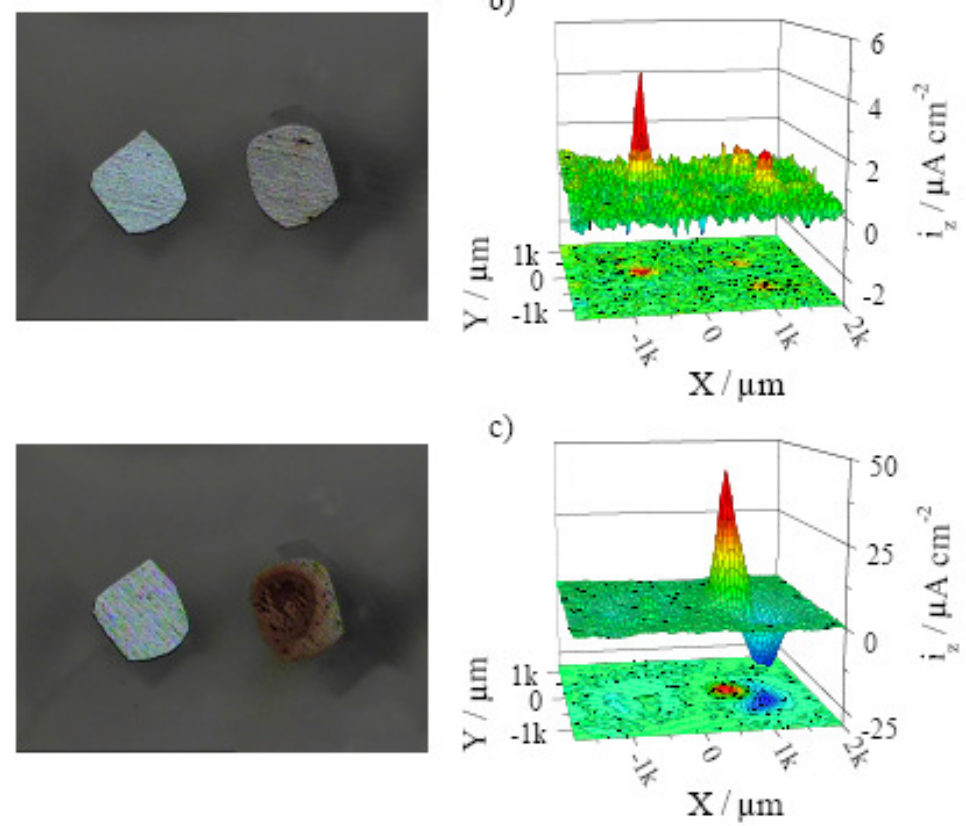

Figure 2. Sample images and SVET current maps obtained in solution above zinc and iron after 24 hours of immersion in the three tested solutions: $0.05 \mathrm{M} \mathrm{NaCl}$ (a), $0.05 \mathrm{M}$ $\mathrm{NaCl}+0.5 \mathrm{~g} \mathrm{~L}^{-1} \mathrm{Ce}\left(\mathrm{NO}_{3}\right)_{3}$ (b), and $0.05 \mathrm{M} \mathrm{NaCl}+0.5 \mathrm{~g} \mathrm{~L}^{-1}$ benzotriazole (c).

\section{SVET}

Both metals corrode in $\mathrm{NaCl}$. However, the corrosive attack is greatly reduced by addition of $\mathrm{Ce}$ (III). When BTA is used as inhibitor, Fe corrodes and $\mathrm{Zn}$ shows very little attack. The inhibition efficiency can be calculated according to Equation 2 where $i$ is the current of sample in the inhibiting medium and $i_{0}$ is the current measured in the corrosive medium. Different ways to obtain $i$ and $i_{0}$ from SVET maps are:
(a) maximum height of anodic peak,
(b) maximum height of cathodic peak,
(c) integration of anodic currents,
(d) integration of cathodic currents,
(e) overall activity in electrode (absolute value of all current detected in measurement).

Table 2 shows the currents determined by the different ways and the corresponding inhibitive efficiency. It also compares the inhibition efficiency estimated by EIS and by SVET. Assuming the efficiency calculated by EIS as the true value, the estimation deviation using SVET, ED, is calculated by, 


$$
E D(\%)=\frac{E I_{S V E T}-E I_{E I S}}{E I_{E I S}} \times 100
$$

\section{Discussion}

The closest estimations given by EIS and SVET were obtained for the cases where inhibition was significant, i.e., for the inhibition with $\mathrm{Ce}$ (III) and for zinc with both inhibitors. In general, the efficiency given by SVET was lower than that given by EIS. At the moment it is difficult to decide for the way of obtaining the SVET currents that lead to the best estimations. Based on the present results, the anodic peak maximum (a), the cathodic peak maximum (b) and the integration of the cathodic activity (d) lead to the closer estimations. Problems will appear in a) and b) if not one but various anodic or cathodic peaks appear. In this case it would be necessary to decide to use, either the value of the maximum peak or the average of all peaks.

Table 2 shows that large deviations were found for iron in BTA. The inhibition efficiency was negative, as if BTA favoured the corrosion of iron. In fact, iron corrosion in $\mathrm{NaCl}$ at near neutral $\mathrm{pH}$ generates insoluble corrosion products that give an underestimation of the current, as described below. Thus, the currents for iron in $\mathrm{NaCl}$ appear lower than the currents in BTA, leading to the observed errors.

The underestimation had less impact with $\mathrm{Ce}$ (III) because in this case the currents were even smaller compared to the uninhibited situation. Zinc in $\mathrm{NaCl}$ produced corrosion products but in a lesser amount compared to Fe and so the errors were proportionally much smaller.

Some difficulties can be encountered regarding the use of SVET, since the ionic currents detected are only those that reach the probe, which is vibrating $200 \mu \mathrm{m}$ above the surface. When the corrosion activity is weak, the ionic fluxes may not attain that distance at a magnitude high enough to be distinguished from the background noise. The same occurs when the anodic and cathodic sites are in big number, very small in size and evenly distributed through the sample area. The current paths between the anodic and cathodic sites will be close to the surface and electro-neutrality in solution will be established just a few microns above the surface. Also, the existence of a layer of corrosion products can hinder the ionic fluxes and make difficult its detection. More important, the formation of corrosion products introduces an underestimation of the current that should be measured. A fraction of the ions generated at the metal surface due to the corrosion process, precipitates before detection by the SVET probe and gives an underestimation of the true current.

Results show that, in optimal conditions, SVET can give accurate inhibition efficiency. However, in other conditions, gross errors can be achieved. Analysis must be done case by case. The images acquired in every SVET measurement can anticipate the success of the inhibition efficiency calculated by SVET. At this stage, it is clear that SVET measurements can provide quantitative information on inhibition efficiency but the technique cannot completely replace the traditional ways like EIS, polarization curves or mass loss determination. 
Table 2. Currents obtained from the SVET maps by different ways: maximum height of anodic peak (a), maximum height of cathodic peak (b), integration of anodic currents (c), integration of cathodic currents (d), overall activity in electrode (e). Also included are the corresponding inhibitive efficiency (IE) and the estimation deviation compared to EIS (ED).

\begin{tabular}{|c|c|c|c|c|c|c|c|c|c|c|c|c|c|c|c|c|}
\hline \multirow[t]{2}{*}{ Metal } & \multirow[t]{2}{*}{ Solution } & \multicolumn{3}{|c|}{ a) } & \multicolumn{3}{|c|}{ b) } & \multicolumn{3}{|c|}{ c) } & \multicolumn{3}{|c|}{ d) } & \multicolumn{3}{|c|}{ e) } \\
\hline & & $\begin{array}{c}i \\
\left(\mu \mathrm{A} \mathrm{cm}^{-2}\right)\end{array}$ & $\begin{array}{l}I E \\
(\%)\end{array}$ & $\begin{array}{l}E D \\
(\%)\end{array}$ & $\begin{array}{c}i \\
\left(\mu \mathrm{A} \mathrm{cm}^{-2}\right)\end{array}$ & $\begin{array}{c}I E \\
(\%)\end{array}$ & $\begin{array}{l}E D \\
(\%)\end{array}$ & $\begin{array}{c}i \\
\left(\mu \mathrm{A} \mathrm{cm}^{-2}\right)\end{array}$ & $\begin{array}{c}I E \\
(\%)\end{array}$ & $\begin{array}{l}E D \\
(\%)\end{array}$ & $\begin{array}{c}i \\
\left(\mu \mathrm{A} \mathrm{cm}^{-2}\right)\end{array}$ & $\begin{array}{c}I E \\
(\%)\end{array}$ & $\begin{array}{l}E D \\
(\%)\end{array}$ & $\begin{array}{c}i \\
\left(\mu \mathrm{A} \mathrm{cm}^{-2}\right)\end{array}$ & $\begin{array}{c}I E \\
(\%)\end{array}$ & $\begin{array}{l}E D \\
(\%)\end{array}$ \\
\hline \multirow[t]{3}{*}{$\mathrm{Fe}$} & $\mathrm{NaCl}$ & 23 & 0 & - & -16 & 0 & - & 1717 & 0 & - & -1329 & 0 & - & 3046 & 0 & - \\
\hline & BTA & 43 & -84.3 & -730 & -19 & -19.2 & -243 & 1470 & 14.4 & +7 & -1730 & -30.3 & -326 & 3201 & -5.1 & -138 \\
\hline & $\mathrm{Ce}$ & 2 & 91.8 & -4.5 & -1 & 93.6 & -2.7 & 396 & 76.9 & -20 & -28 & 97.9 & +2 & 424 & 86.1 & -10.5 \\
\hline \multirow[t]{3}{*}{$\mathrm{Zn}$} & $\mathrm{NaCl}$ & 64 & 0 & - & -26 & 0 & - & 3490 & 0 & - & -2964 & 0 & - & 6454 & 0 & - \\
\hline & BTA & 4 & 95.0 & -4.7 & -4 & 85.2 & -14.4 & 273 & 92.2 & -7.4 & -550 & 81.4 & -18.2 & 823 & 87.2 & -12.4 \\
\hline & $\mathrm{Ce}$ & 5 & 93.1 & -6.2 & -1 & 96.4 & -2.8 & 398 & 88.6 & -10.7 & -33 & 98.9 & -0.3 & 431 & 93.3 & -5.9 \\
\hline
\end{tabular}




\section{Conclusions}

The use of SVET to obtain the efficiency of corrosion inhibitors was analysed. Good correlation was encountered for high levels of inhibition. Discrepancies existed when corrosion products were formed, leading to an underestimation of the current put in play in the processes. Visual inspection of the samples may identify the cases more susceptible to deviation. The approach discussed here can be used to obtain more quantitative information from SVET measurements but should not replace the traditional ways used to estimate the inhibition efficiency, like EIS, polarization curves or mass loss determination.

\section{Acknowledgments}

A. C. Bastos and M. L. Zheludkevich thank Fundação para a Ciência e a Tecnologia for post-doc grants.

\section{References}

1. H.H. Uhlig, Ed., The Corrosion Handbook, J. Wiley \& Sons, New York, 1948, p. 909.

2. E. Barsoukov, J.R. MacDonald, Eds., Impedance Spectroscopy, $2^{\text {nd }}$ ed., Wiley Interscience, New Jersey, 2005, p. 344.

3. O. Bluh, B. Scott, Rev. Sci. Instr. 21 (1950) 867.

4. $\quad$ L.F. Jaffe, R. Nuccitelli, J. Cell Biol. 63 (1974) 614.

5. $\quad$ P. Somieski, W. Nagel, J. Exp. Biol. 201 (1998) 2489.

6. H.S. Isaacs, G. Kissel, J. Electrochem. Soc. 119 (1972) 1628.

7. H.S. Isaacs, Y. Ishikawa, in Electrochemical Techniques for Corrosion Engineering, R. Baboian, Ed., NACE, Houston, 1986, p. 22.

8. H.S. Isaacs, Corros. Sci. 28 (1988) 547.

9. K. Ogle, V. Baudu, L. Garrides, X. Philippe, J. Electrochem. Soc. 147 (2000) 3654.

10. J.L. Yao, B. Ren, Z.F. Huang, P.G. Cao, R.A. Gu, Zhong-Qun Tian, Electrochim. Acta 48 (2003) 1263.

11. B.R.W. Hinton, L. Wilson, Corros. Sci. 29 (1989) 967. 